\title{
Reflets
}

Revue ontaroise d'intervention sociale et communautaire

\section{Un Témoignage - le profil de Philippe}

\section{Julie Joanisse}

Volume 7, numéro 1, printemps 2001

Santé mentale et les défis de l’an 2001

URI : https://id.erudit.org/iderudit/026345ar

DOI : https://doi.org/10.7202/026345ar

Aller au sommaire du numéro

Éditeur(s)

Reflets : Revue ontaroise d'intervention sociale et communautaire

ISSN

1203-4576 (imprimé)

1712-8498 (numérique)

Découvrir la revue

Citer cet article

Joanisse, J. (2001). Un Témoignage - le profil de Philippe. Reflets, 7(1), 200-203. https://doi.org/10.7202/026345ar

Tous droits réservés (C) Reflets : Revue ontaroise d'intervention sociale et communautaire, 2001
Ce document est protégé par la loi sur le droit d'auteur. L'utilisation des services d'Érudit (y compris la reproduction) est assujettie à sa politique d'utilisation que vous pouvez consulter en ligne.

https://apropos.erudit.org/fr/usagers/politique-dutilisation/ 


\section{Un Témoignage - le profil de Philippe}

\section{Julie Joanisse}

Philippe est un jeune homme de 17 ans. Il possède une humeur très variable avec un bon sens de l'humour. Il adore jaser et être en compagnie des gens. Ses manières d'agir ne sont pas celles d'un adolescent de 17 ans. Par exemple, lorsqu'il reçoit un cadeau, il s'excite facilement. Depuis plusieurs années, il souffre d'une maladie mentale. Cette maladie, diagnostiquée comme étant «maniaco-dépressive», est causée par un déséquilibre chimique du cerveau. Chaque personne connait des moments heureux et d'autres où elle est plus déprimée. Cependant, pour Philippe, le transfert entre ces états émotifs est beaucoup plus rapide et les sentiments sont beaucoup plus dramatiques. Quand Philippe a une saute d'humeur, par exemple, lorsqu'il est content, il devient alors surexcité. Cependant, quand il se sent malheureux, il devient très rapidement dépressif. Ainsi, Philippe déborde les deux extrémités dans la gamme des émotions.

Philippe vit avec cette maladie depuis qu'il est jeune. Cependant, il n'a été diagnostiqué qu'à l'âge de onze ans. Depuis cet âge, Philippe a passé beaucoup de temps à l'hôpital.

Aujourd'hui, sa maladie est rendue tellement grave qu'il entend des voix et, à l'occasion, il hallucine. Après plusieurs épisodes agressifs, mes parents ont dû prendre la décision de le placer dans une institution. Les professionnels de la santé dans cette institution sont sensés lui montrer comment contrôler ses épisodes agressifs et réduire l'effet des voix. 


\section{Les effets sur la famille}

Je viens d'une famille recomposée, où chaque parent arrive d'un mariage précédent.J'ai un frère biologique, Marc, une soeur, Mélisse, et un frère Philippe. Mélisse et Philippe sont les enfants nés de l'épouse de mon père.Au début de ce deuxième mariage, c'était très difficile. Philippe était agressif. Par exemple, il a défoncé les murs en faisant plusieurs trous. Mon père était toujours frustré et fâché. Marc et moi sentions que c'était très injuste de vivre avec quelqu'un qui était toujours dans le trouble. Je me rappelle que mes parents essayaient de porter de l'attention à chacun de nous, mais en réalité, Philippe prenait la plupart de leur temps. À chaque semaine, nous devions faire une rencontre de famille pour régler les chicanes entre Philippe et les trois autres enfants. À chaque jour, mes parents devaient discipliner Philippe. Je me rappelle me sentir mal pour ma soeur car Marc et moi vivions avec ma mère, donc nous n'étions pas toujours là quand Philippe faisait des bêtises et mon père se fâchait.

Lorsque Philippe a été diagnostiqué, la famille ne comprenait pas la maladie et ne savait pas comment la contrôler. Au début, Philippe a dû rester à l'hôpital Algoma pour se faire examiner. D'une façon, la famille se sentait soulagée qu'il n'était pas avec nous. Nous sentions que nos parents avaient finalement du temps pour nous. Après quelque temps, nos sentiments ont encore changé. Encore une fois, Philippe est devenu le centre d'attention de mes parents et nous devions mettre nos besoins de côté. Les professionnels de la santé de l'hôpital Algoma ont alors décidé qu'ils voulaient rencontrer toute la famille. Donc, à chaque deux semaines, il fallait voyager de Timmins à Sudbury pour parler aux médecins. Nous avons dû participer à des sessions pour parler de Philippe et discuter de nos sentiments. Au début, je détestais cette situation, car à chaque fin de semaine, quand j'allais chez mon père, il fallait toujours passer à Sudbury pour Philippe. Je sais que mon frère et ma soeur se sentaient de la même façon. Nous étions las d'entendre parler de Philippe. Nous étions fatigués 
d'avoir à voyager pour lui à Sudbury. Nous voulions seulement passer du temps avec nos parents, sans Philippe et sans devoir parler de lui. Les rencontres à l'hôpital servaient à nous faire comprendre ce qui se passait avec Philippe et à nous aider à supporter ses sautes d'humeur et son agressivité. Aujourd'hui, je pense que les sessions nous ont vraiment aidés. Je pense que sans les sessions, notre famille se serait écroulée. Je suis contente que le psychiatre, les psychologues et mes parents nous ont aidés à comprendre ce qui se passait. Tout au long de ce séjour, mes parents ont vraiment essayé de nous appuyer et de nous faire comprendre ce qui se passait vraiment.

\section{Les effets du diagnostic et comment la famille et Philippe vivent maintenant}

Je pense que l'effet du diagnostic a d'abord fait une différence dans la vie de mes parents. Mes parents ont dû pour la première fois sentir un soulagement. Ils ne se sentaient plus comme s'ils étaient de méchants parents. Enfin, ils savaient que Philippe n'était pas un enfant malin mais malade. Ce n'était ni la faute de mes parents, ni la faute de Philippe. Je pense qu'auparavant, il y avait beaucoup de sentiments de culpabilité. Cependant, lorsque le psychiatre a fait son diagnostic, ces sentiments ont beaucoup diminué. Pour les enfants, au début, le diagnostic ne nous disait rien. Cependant, avec le temps et de l'aide, nous avons pu mieux comprendre ce qui se passait.

Aujourd'hui, Philippe vit dans une institution. Il nous dit toujours que c'est une prison et qu'il veut revenir à la maison. Nous essayons de lui dire qu'il n'est pas prêt, qu'il n'a pas appris les techniques nécessaires pour contrôler les voix dans sa tête. Cependant, souvent, il ne comprend pas. Pour Philippe, il ne fait souvent pas partie de la réalité. C'est-à-dire que pour Philippe, les voix qu'il entend dans sa tête prennent le contrôle. Ces voix occupent donc environ $90 \%$ de sa réalité. Quand Philippe est capable de voir ou de comprendre notre réalité, il sait qu'il est très malade et qu'il doit rester dans l'institution. 
Je peux dire que la famille a beaucoup de peine à le voir aussi déprimé et à le voir dans une place pareille. Par contre, nous savons qu'il a besoin de contrôler ses épisodes agressifs. À chaque soir, il y a au moins un membre de la famille qui lui téléphone. Parfois, chaque membre de la famille prend le temps de lui parler. Je pense que ceci est très important pour chacun de nous, surtout pour Philippe. Cette habitude lui dit que nous l'aimons.

\section{Le monde professionnel}

Au début, quand nous savions qu'il y avait quelque chose de mal avec Philippe, les médecins nous disaient non, que c'était seulement son attitude. Ils disaient que Philippe était méchant ou que mes parents ne faisaient rien pour le discipliner.Lorsque l'hôpital Algoma s'est impliqué, le psychiatre, les travailleurs sociaux et les psychologues nous ont vraiment aidés. Philippe a fréquenté l'hôpital plusieurs fois au cours d'une période de cinq ans. Lorsque ses épisodes agressifs ou dépressifs devenaient trop graves, il devait y retourner.

Je trouve qu'il y a peu de ressources dans la communauté pour les jeunes qui ont des problèmes de santé mentale. Lorsque mon frère était adolescent, nous étions toujours confrontés à des obstacles. Philippe ne pouvait pas aller à l'école secondaire comme les autres adolescents de son âge. Il ne pouvait pas tenir des emplois à temps partiel et il entendait des voix que la plupart des autres jeunes de son âge n'entendaient pas. La communauté n'offre encore aucun programme pour les adolescents qui souffrent de maladies mentales. Par conséquent, Philippe a été abandonné par le système.

Maintenant qu'il est presque adulte, il y aura plus de programmes et de ressources dont il pourra profiter.

N.B. Il est triste de constater que les besoins des adolescents tombent dans l'indifférence d'une société qui a développé des services pour enfants et pour adultes en oubliant de prendre soin de ces adolescents souffrant de maladie mentale. On nous dit qu'il manque de ressources financières. Est-ce vraiment le cas? 